2004-01-01

\title{
Power Coupling Between Two Step-Index Multimode Fibers of Different Numerical Apertures with an Angular Misalignment
}

Cheng Gao

Dublin Institute for Advanced Studies

Gerald Farrell

Technological University Dublin, gerald.farrell@tudublin.ie

Follow this and additional works at: https://arrow.tudublin.ie/engscheceart

Part of the Electrical and Computer Engineering Commons

\section{Recommended Citation}

Gao, C., Farrell, G.:Power Coupling Between Two Step-Index Multimode Fibers of Different Numerical Apertures with an Angular Misalignment. Microwave and Optical Technology Letters, Vol.43, no.3, 2004, pp.231-234

This Article is brought to you for free and open access by the School of Electrical and Electronic Engineering at ARROW@TU Dublin. It has been accepted for inclusion in Articles by an authorized administrator of ARROW@TU Dublin. For more information, please contact arrow.admin@tudublin.ie, aisling.coyne@tudublin.ie, gerard.connolly@tudublin.ie.

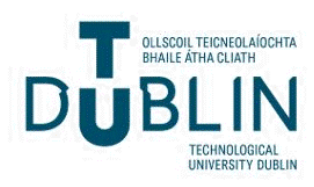


The paper is accepted and will be published in the Journal of Microwave and Optics Technology Letters in November 2004 


\title{
Power coupling between two step-index multimode fibers of different numerical apertures with an
} angular misalignment

\author{
Cheng Gao, Gerald Farrell
}

School of Electronics and Communications

Faculty of Engineering

Dublin Institute of Technology

Kevin Street, D8

Dublin, Ireland

Abstract-Coupling efficiency between two stepindex multimode fibers of different numerical apertures with an angular misalignment is calculated. The theory is presented using radiometry and geometrical optics. The limit case of the calculation is consistent with a previously published result. The results may be useful for applications such as power launching between two types of fibers with different numerical apertures for example silica fiber and plastic optical fiber (POF) and with an angular misalignment.

coupling efficiency, power launching, silica fiber, POF, numerical aperture, angular misalignment, geometrical optics.

\section{INTRODUCTION}

Losses occur when jointing two optical fibers by a splice or a connector. They fall into a number of categories. Intrinsic losses are caused by mismatched fibers with different numerical apertures, core diameters or index profiles for the fibers being jointed. Previous publications have derived exact theories for intrinsic losses [1], [2]. Extrinsic losses are caused by geometrical misallignment of the fiber ends. An exact theory exists in the case of a radial offset [3] and tilt [4] whereas an approximation exists in the case of an air gap [5]. Fresnel reflection losses are caused by the refractive index differences between fiber cores and an air gap [6], [7].

Analysis and results of power coupling between two identical fibers with angular misalignment has existed in a previous publication [4], but for the case of fibers

\section{Definitions And Assumptions}

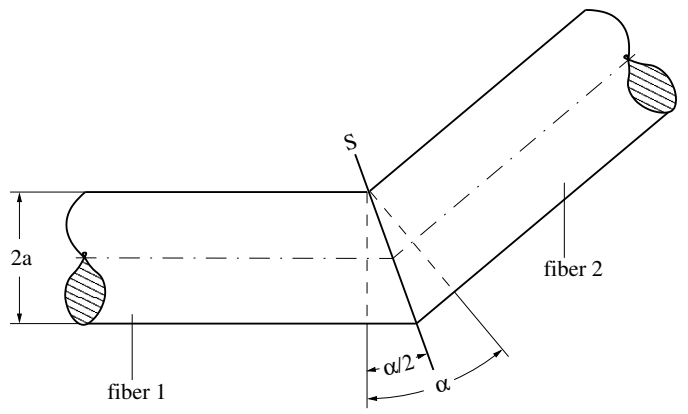

Fig. 1. Definitions.

with both angular misalignment and different numerical apertures there exists no analysis so far. In this paper, we discuss the power coupling between two step-index fibers with a same core diameter but with different numerical apertures. The theory of coupling efficiency is presented using radiometry and geometrical optics.

The essential graphic definitions could be given in Fig.1. Fiber 1 is the transmitting fiber, fiber 2 is the receiving fiber, meeting at a contact plane $S$. Furthermore we assume that

1) both fibers are multimode step-index fiber with the same core size,

2) $\mathrm{NA}_{1} \leq \mathrm{NA}_{2}$, which is a specific case we analysed in this paper, where $\mathrm{NA}_{1}$ and $\mathrm{NA}_{2}$ is the numerical aperture of fiber 1 and 2, respectively.

3) a uniform mode power distribution in the misalignment plane $S$,

4) leaky modes are to be neglected,

5) the integration has to be taken over the entire fiber core contact plane $S$. 


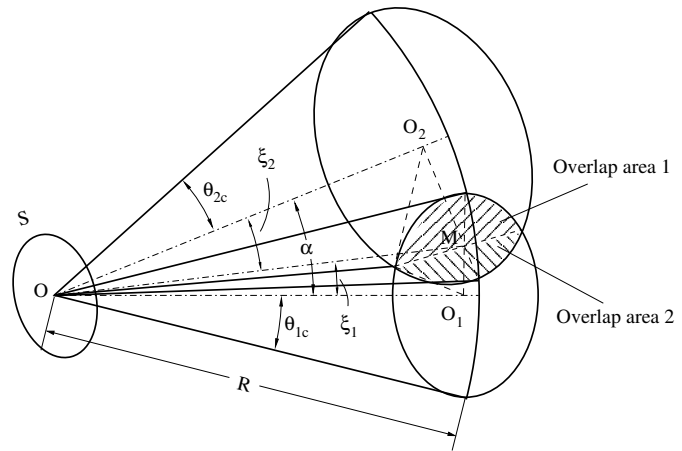

(a)

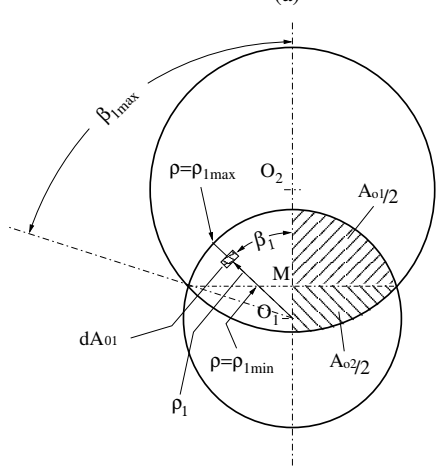

(b)

Fig. 2. Overlapping Cones.

\section{Calculation And Results}

The coupling efficiency of power launching from fiber 1 to fiber 2 can be determined from the ratio of transmitted power within fiber 2 over the total launched power within fiber 1 . The total power within fiber 1 for stepindex profile is written as [8, p217]

$$
P_{\mathrm{si}}=\pi^{2} a^{2} L(\mathrm{NA})^{2}
$$

where $P_{\mathrm{si}}$ is the total power of step-index multimode fiber, for simplicity, the subscript si which indicates step-index profile will be omitted in the subsequent discussion, $L$ is the radiance of the light source, which is also the radiance of the endface of receiving fiber and NA is the numerical aperture of the fiber.

The transmitted power within fiber 2 can be calculated by integrating within the plane $S$ over the solid angle in the overlap area of the acceptance cones of fiber 1 and fiber 2, as shown in Fig.2. The overlap area consists of two areas designated as overlap area 1 and 2 for fiber 1 and fiber 2, respectively. Because the two fibers have different numerical apertures, which means the acceptance cones of the two fibers are not identical, then the overlap area 1 is not identical to the overlap area
2. Therefore, the integration over the two overlap areas must be solved individually and the overall integration can be calculated by adding the integrate power over overlap area 1 and 2 .

We designate the integration within the overlap area 1 and 2 as $P_{21}$ and $P_{22}$, respectively, which also means the transmitted power within fiber 1 and 2 , respectively. The overall transmitted power within fiber $2, P_{2}$, is the sum of the transmitted power within overlap area 1 and 2 . Thus

$$
P_{2}=P_{21}+P_{22}
$$

The integration over overlap area 1 can be solved by integrating over the area element $\mathrm{d} A_{\mathrm{o} 1}$ of overlap area 1 , within the integration limit of angle $\beta_{1}$ and radius $\rho_{1}$, as shown in Fig.2.

In general the total power emitted by a light source is written as

$$
P=\int_{\Omega} \int_{S} L \cos \theta \mathrm{d} \Omega \mathrm{d} S
$$

where $\Omega$ is the solid angle over which the radiation extends, $S$ the area of the emitting surface and $\theta$ is the angle between the radiation extent and the normal of the emitting surface. The power density of a light source is written as the inner integration of Eq.3

$$
P^{\prime}=\int_{\Omega} L \cos \theta \mathrm{d} \Omega
$$

For the transmitted power in fiber 2 within overlap area 1 , the power density can be written as

$$
P_{21}^{\prime}=\int_{\Omega_{21}} L_{\mathrm{s}} \cos \theta_{\mathrm{s}} \mathrm{d} \Omega_{21}
$$

where $L_{\mathrm{s}}$ is the radiance based on the power distribution in the plane $S, \Omega_{21}$ is the solid angle of fiber 2 within overlap area 1 , and $\theta_{\mathrm{S}}$ is the angle between the radiation extent within overlap area 1 and the normal of contact plane $S$.

We first evaluate the $P_{21}^{\prime}$ using the given parameters in Fig.2. Corresponding to the definition of the solid angle $\Omega$, within overlap area 1 :

$$
\Omega_{21}=\frac{A_{\mathrm{o} 1}}{R^{2}}
$$


where $R$ is the radius of the acceptance cone for both fiber 1 and fiber 2. Furthermore, Eq. 6 can be expanded as:

$$
\Omega_{21}=\frac{2}{R^{2}} \int_{\beta_{1}=0}^{\beta_{1 \max }} \int_{\rho_{1 \min }}^{\rho_{1 \max }} \mathrm{d} A_{\mathrm{o} 1}
$$

where $\beta_{1 \text { max }}$ is the maximum angle within overlap area 1 , the minimum angle of $\beta_{1}$ is zero as is obvious in Fig.2, $\rho_{1 \text { max }}$ and $\rho_{1 \text { min }}$ is the maximum and minimum radius of $\rho_{1}$, where the area element $\mathrm{d} A_{\mathrm{o} 1}$ can be described as

$$
\mathrm{d} A_{\mathrm{o} 1}=R^{2} \sin \theta_{1} \mathrm{~d} \theta_{1} \mathrm{~d} \beta_{1}
$$

$\theta_{1}$ is the angle between the radiation extent and the cone axis $\mathrm{OO}_{1}$ of fiber 1 .

The integration limits of $\beta_{1}$ and $\rho_{1}$ can be evaluated by first evaluating the angles $\xi_{1}$ and $\xi_{2}$, which are the angles of cone axis $\mathrm{OO}_{1}$ and $\mathrm{OO}_{2}$ to the intersection plane between overlapping cones and are also the angles between $\mathrm{OO}_{1}, \mathrm{OO}_{2}$ and $\mathrm{OM}$, respectively, as shown in Fig.2. They are related to the angle of misalignment and the acceptance angle of the two cones as

$$
\begin{aligned}
\xi_{1}+\xi_{2} & =\alpha \\
\mathrm{OM} \cos \xi_{1} & =R \cos \theta_{1 \mathrm{c}} \\
\mathrm{OM} \cos \xi_{2} & =R \cos \theta_{2 \mathrm{c}}
\end{aligned}
$$

where $\theta_{1 \mathrm{c}}$ and $\theta_{2 \mathrm{c}}$ is the acceptance angle of fiber 1 and fiber 2, respectively. Solving Eq. 9, 10 and 11 gives

$$
\begin{aligned}
\xi_{1} & =\arctan \left(\frac{\cos \theta_{2 c}}{\cos \theta_{1 c} \sin \alpha}-\frac{1}{\tan \alpha}\right) \\
\xi_{2} & =\arctan \left(\frac{\cos \theta_{1 c}}{\cos \theta_{2 c} \sin \alpha}-\frac{1}{\tan \alpha}\right)
\end{aligned}
$$

Furthermore,

$$
\begin{aligned}
& \mathrm{O}_{1} \mathrm{M}=R \sin \theta_{1 \mathrm{c}} \cos \beta_{1 \max } \\
& \mathrm{O}_{1} \mathrm{M}=R \cos \theta_{1 \mathrm{c}} \tan \xi_{1}
\end{aligned}
$$

as is shown in Fig.2. Solving Eq.14 and 15 gives the maximum value of angle $\beta_{1}$ of

$$
\beta_{1 \max }=\arccos \left(\frac{\sin \xi_{1}}{\sin \theta_{1 c}}\right)
$$

The minimum and maximum value of $\rho_{1}$ is given by:

$$
\begin{aligned}
\rho_{1 \min } & =R \frac{\sin \xi_{1}}{\cos \beta} \\
\rho_{1 \max } & =R \sin \theta_{1 c}
\end{aligned}
$$

with $\sin \theta_{1}=\rho_{1} / R$, we obtain the limit of the inner integral of Eq.7:

$$
\begin{aligned}
\sin \theta_{1 \min } & =\frac{\sin \xi_{1}}{\cos \beta} \\
\sin \theta_{1 \max } & =\sin \theta_{1 c}
\end{aligned}
$$

The power density $P_{21}^{\prime}$ in fiber 2 within overlap area 1 is thus

$$
P_{21}^{\prime}=2 \int_{\beta_{1}=0}^{\beta_{1 \max }} \int_{\theta_{1 \min }}^{\theta_{1 \max }} L_{\mathrm{s}} \cos \theta_{\mathrm{s}} \sin \theta_{1} \mathrm{~d} \theta_{1} \mathrm{~d} \beta_{1}
$$

The integration becomes sophiscated when $\theta_{\mathrm{S}}$ is involved and the relationship between $\theta_{\mathrm{s}}$ and $\theta_{1}$ has to be determined. However, integrating over contact plane $S$ with a radiance of $L_{\mathrm{s}}$ becomes the same as integrating over a plane perpendicular to the fiber axis with a radiance $L$ of $[8, \mathrm{p} 241]$

$$
L=\frac{L_{\mathrm{s}}}{\cos \alpha / 2}
$$

Thus, integrating $P_{21}^{\prime}$ over contact plane $S$ with a radiance $L_{\mathrm{s}}$ can be solved by integrating $P_{21}^{\prime}$ over the endface of the fiber 1 with a radiance $L$.

By rewritting Eq.21 using $L$ and $\theta_{1}$ to replace $L_{\mathrm{s}}$ and $\theta_{\mathrm{s}}$, respectively, we obtain:

$$
P_{21}^{\prime}=2 \int_{\beta_{1}=0}^{\beta_{1 \max }} \int_{\theta_{1 \min }}^{\theta_{1 \max }} L \cos \theta_{1} \sin \theta_{1} \mathrm{~d} \theta_{1} \mathrm{~d} \beta_{1}
$$

The integral of Eq.23 can be solved as

$$
P_{21}^{\prime}=L\left(\sin ^{2} \theta_{1 \mathrm{c}} \beta_{1 \text { max }}-\sin ^{2} \xi_{1} \tan \beta_{1 \text { max }}\right)
$$

using Eq.16, 19 and 20.

Using an identical approach, the integration over solid angle of fiber 2 within the overlap area 2 yields the power density:

$$
P_{22}^{\prime}=L\left(\sin ^{2} \theta_{2 \mathrm{c}} \beta_{2 \max }-\sin ^{2} \xi_{2} \tan \beta_{2 \max }\right)
$$


with the integration limit:

$$
\beta_{2 \max }=\arccos \left(\frac{\sin \xi_{2}}{\sin \theta_{2 \mathrm{c}}}\right)
$$

The transmitted power in fiber 2 within overlap area 1 is found by integrating $P_{21}^{\prime}$ over the endface of fiber 1 as is stated above, which is simply the power density, $P_{21}^{\prime}$, multiplied by the area of the endface, thus

$$
\begin{aligned}
P_{21} & =\pi a^{2} P_{21}^{\prime} \\
& \left.=\pi a^{2} L\left[\beta_{1 \max } \mathrm{NA}_{1}^{2}-\sin ^{2} \xi_{1} \tan \beta_{1 \max }\right] 28\right)
\end{aligned}
$$

where $\mathrm{NA}_{1}\left(=\sin \theta_{1 \mathrm{c}}\right)$ and $\mathrm{NA}_{2}\left(=\sin \theta_{2 \mathrm{c}}\right)$ are the numerical apertures of fiber 1 and fiber 2 , respectively.

In the same way, the transmitted power in fiber 2 within overlap area 2 can be found as:

$$
\begin{aligned}
P_{22} & =\pi a^{2} P_{22}^{\prime} \\
& =\pi a^{2} L\left[\beta_{2 \max } \mathrm{NA}_{2}^{2}-\sin ^{2} \xi_{2} \tan \beta_{2 \max }\right](30)
\end{aligned}
$$

The total power within fiber 1 is

$$
P_{1}=\pi^{2} a^{2} L \mathrm{NA}_{1}^{2}
$$

as is in Eq.1. The overall transmitted power in fiber 2, $P_{2}$, is the sum of $P_{21}$ and $P_{22}$ as shown in Eq.2. The coupling efficiency is the ratio of the overall transmitted power within fiber 2 to total power within fiber 1 , thus

$$
\eta=\frac{P_{2}}{P_{1}}
$$

Finally using Eq.2, Eq.28, Eq.30 and Eq.31, the coupling efficiency is given by:

$$
\begin{aligned}
\eta & =\frac{1}{\pi \mathrm{NA}_{1}^{2}}\left[\beta_{1 \max } \mathrm{NA}_{1}^{2}-\sin ^{2} \xi_{1} \tan \beta_{1 \max }\right] \\
& +\frac{1}{\pi \mathrm{NA}_{2}^{2}}\left[\beta_{2 \max } \mathrm{NA}_{2}^{2}-\sin ^{2} \xi_{2} \tan \beta_{2 \max }\right]
\end{aligned}
$$

In Fig. 3 the coupling efficiency $\eta$ is depicted as a function of $\sin \alpha / \sin \theta_{1 \mathrm{c}}$ at four different ratio $r$ of $\sin \theta_{2 \mathrm{c}} / \sin \theta_{1 \mathrm{c}}$. The coupling efficiency can be plotted as a function of the angle of misalignment. For simplicity we use a

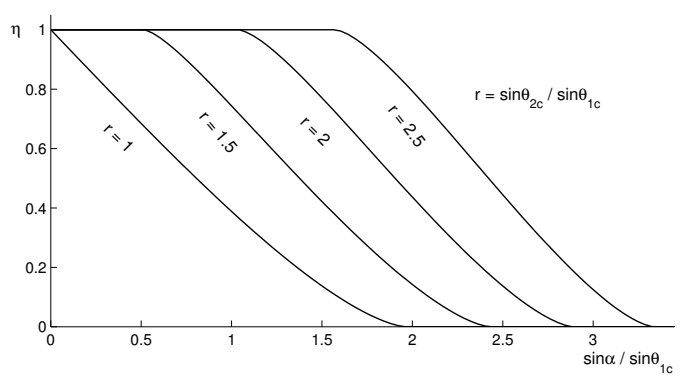

Fig. 3. Coupling efficiency.

normalized angle of misalignment, which is the ratio of the sine value of $\alpha$ to the sine value of $\theta_{1 \mathrm{c}}$. The coupling efficiency is plotted as a function of the ratio, $r$, the ratio of the numerical apertures of the two fibers.

\section{Discussion}

\section{A. Solution for $r>1$}

For any $r>1$, because $r=\sin \theta_{2 c} / \sin \theta_{1 c}$, the acceptance angle of fiber 2 is greater than that of fiber 1 . A small tilt angle between the axes of the two fibers will not result in loss occuring since the acceptance cone of fiber 1 remains inside the acceptance cone of fiber 2 . All the optical power will be coupled from fiber 1 into fiber 2 and the coupling efficiency is 1 . In Fig.3, the top horizontal line indicates a unity coupling efficiency and the corresponding angle of misalignment $\alpha$ is in the range $\left[0, \theta_{2 \mathrm{c}}-\theta_{1 \mathrm{c}}\right]$, which can thus be designated as a zero-loss region. For a constant value of $\theta_{1 \mathrm{c}}$, because $r=\sin \theta_{2 \mathrm{c}} / \sin \theta_{1 \mathrm{c}}$, a larger $r$ will represent a larger zero-loss region since a larger $r$ indicates a larger $\theta_{2 c}$ and thus a larger difference $\theta_{2 \mathrm{c}}-\theta_{1 \mathrm{c}}$. A larger zero-loss region means that the coupling efficiency is tolerant of the angle of misalignment.

Where loss does occur, the tilt angle $\alpha$ lies within the range $\left[\theta_{2 \mathrm{c}}-\theta_{1 \mathrm{c}}, \theta_{2 \mathrm{c}}+\theta_{1 \mathrm{c}}\right]$, so that the acceptance cone of fiber 1 is partially outside the acceptance cone of fiber 2. Furthermore at any specific angle of misalignment the coupling efficiency will be larger at a larger value of $r$, thus the coupling efficiency increases with the increases in $r$.

Finally, in the case where $\alpha$ is greater than $\theta_{2 \mathrm{c}}+\theta_{1 \mathrm{c}}$, there is no overlap between acceptance cones of the two fibers and no optical power will be coupled from fiber 1 into fiber 2. The coupling efficiency is thus zero as indicated in the flat tail of each curve in Fig.3. 


\section{B. Solution for $r=1$}

For comparison with previous result, when $r=1$, the solution becomes the calculation of coupling efficiency between two step-index fibers with identical numerical apertures. Thus,

$$
\theta_{2 c}=\theta_{1 c}
$$

Combined with Eq.12 and 13, we can derive:

$$
\xi_{1}=\xi_{2}=\alpha / 2
$$

Using Eq.34, Eq.16 and 26, we can further derive:

$$
\beta_{1 \max }=\beta_{2 \max }=\arctan \left(\frac{\sin ^{2} \theta_{1 \mathrm{c}}}{\sin ^{2} \alpha / 2}-1\right)^{1 / 2}
$$

Solving Eq.33, 35 and 36, the coupling efficiency is:

$\eta=\frac{2}{\pi}\left[\arctan \left(\frac{\sin ^{2} \theta_{1 \mathrm{c}}}{\sin ^{2} \alpha / 2}-1\right)^{1 / 2}-\frac{\sin ^{2} \alpha / 2}{\sin ^{2} \theta_{1 c}}\left(\frac{\sin ^{2} \theta_{1 \mathrm{c}}}{\sin ^{2} \alpha / 2}-1\right)^{1 / 2}\right]$

which is the exact solution for the case of step-index profile in [4, Eq.28]. Fig.3 also shows the curve of $r=1$ for comparison with other curves of $r>1$.

\section{Conclusion}

The coupling efficiency for a uniform distributed power coupling between two step-index multimode fibers with different numerical apertures and an angular misalignment has been calculated. This may find its application when dealing with power coupling between two types of fibers such as silica fiber and POF with an angular misalignment. The theory is presented using radiometry and geometrical optics. The limit case of the calculation is consistent with a previously published result.

\section{REFERENCES}

[1] S.C.Mettler, Bell Syst.Tech.J.58, 2163 (1979).

[2] E.-G.Neumann, NTZ Arch.2, 159 (1980).

[3] E.-G.Neumann and W.Weidhaas, AEU Arch.fuer Elektron.und Uebertragungstech.Electron.and Commun.30, 448 (1976).

[4] D.Opielka and D.Rittich, 'Transmission loss caused by an angular misalignment between two multimode fibers with arbitrary profile exponents', Appl.Opt. vol. 22, no. 7, April 1983, pp. 991-4.
[5] W.van Etten, W.Lambo and P.Simons, 'Loss in multimode fiber connections with a gap', Appl.Opt. vol. 24, no. 7, April 1985, pp. 970-6.

[6] D.Marcuse, Appl.Opt.14, 3016 (1975).

[7] R.E.Wagner and C.R.Sandahl, 'Interference effects in optical fiber connections', Appl.Opt. vol. 21, no. 8, April 1982, pp. 1381-5.

[8] W. Van Etten, J. Van Der Plaats, Fundamentals of Optical Fiber Communications, (London: Prentice Hall, 1991). 